\title{
Spatial Spline Construction through the Monge Model
}

\author{
Konstantin Panchuk ${ }^{[0000-0001-9302-8560]}$, Tatyana Myasoedova ${ }^{[0000-0002-9641-9417]}$ \\ and Yuri Rogoza ${ }^{[0000-0003-1552-0570]}$ \\ Omsk State Technical University, Mira Ave., 11, 644050, Omsk, Russia \\ panchuk_kl@mail.ru, mtm44mtm44@mail.ru,rogoza_ya@yandex.ru
}

\begin{abstract}
The solution to the problem of spatial spline construction by its orthogonal projections on the Monge model is considered in the present paper. Constructively, spatial interpolation of a discrete set of points given on projection planes $\pi_{1}$ and $\pi_{2}$ is performed through planar interpolation of its projections. The initial boundary conditions for spatial spline construction are given in the form of initial derivative vector projections in the initial and the terminal points of the discrete set. The possibility of the solution to the problem of spatial spline construction by planar projections, i.e. reduction of a spatial solution to a planar one, is determined by the projectional properties of the Monge model. An algorithm of construction of a spatial polynomial segment by projectionally defined initial conditions - projections of two points and projections of the initial derivatives in these points - is considered. A solution to a more complex problem - formation of a spatial spline consisting of a number of segments connected under a certain order of smoothness - is proposed on the basis of this algorithm. The validity of the proposed projectional algorithm of spline formation is confirmed on numerical example. The algorithm can be applied in solution to a more general and relevant problem of synthesis of 3D geometric models by their projectional 2D images that is currently lacking complete solution.
\end{abstract}

Keywords: Spatial Spline, Monge Model, Polynomial Segment.

\section{Introduction}

The commonly known term "spline" is used to describe a geometric image in the shape of a curve defined by the mathematical model of a smooth segment-polynomial function constructed through a specific algorithm. The spline function is an essential instrument of various computational methods widely relied upon in a number of science, engineering, and design applications. The particularly significant ones include design and construction of surface forms in the fields of machine-building, naval architecture, aircraft construction, architecture and construction. Spline is the basic element of surface form generation; it is also applied in solutions to the problems of

Copyright (C) 2020 for this paper by its authors. Use permitted under Creative Commons License Attribution 4.0 International (CC BY 4.0). 
interpolation and smoothing [1-5]. The solutions to surface form generation problems apply both flat and spatial splines. It is known that construction of a spatial spline is more algorithmically complex and computationally heavy than construction of a flat spline. At the same time, there are models of space $R^{3}$ known in the field of engineering geometry, for example, constructive models based on the method of two images and two traces [6]. These models of space realize various spatial theoretical and applied tasks. The Monge model (Monge drawing), as a particular case of the method of two images, constitutes a homeomorphic model of space $R^{3}$, i.e. the property of continuity is the invariant of orthogonal representation [7]. The question arises: Does the Monge model have the capability to realize a model of spatial spline, i.e. is it possible to construct a spatial spline through its planar projections? In order to answer this question, let us refer to the definition of the term "spline". According to the known definition $[1,8,9]$, a spline of order $m$ is a function $\bar{a}(t):[a, b] \rightarrow R^{3}$ of a real variable $t$ defined on a net with real knots $t_{i}: a=t_{0} \leq t_{1} \leq \ldots \leq t_{n}=b$ such that:

1. $\bar{a}(t)$ is a polynomial of order $p \geq 2$ on each segment $\left[t_{i}, t_{i+1}\right], \quad i=0, \ldots, n-1$;

2. $\bar{a}(t)$ is a $C^{p-1}$ function.

It obviously follows from the definition of the term "spline" and the mentioned properties of the Monge model that this model has the capability to realize a model of spatial line:

$$
\bar{a}(t):[a, b] \rightarrow a \subset R^{3} \leftrightarrow\left\{\begin{array}{l}
\bar{a}_{x y}:[a, b] \rightarrow a_{x y} \subset R_{x y}^{2}, \\
\bar{a}_{x z}:[a, b] \rightarrow a_{x z} \subset R_{x z}^{2},
\end{array}\right.
$$

where a, $a_{x y}, a_{x z}$ represents a spatial spline and its orthogonal projections. Therefore, the Monge model (Monge method) is accepted as the method for solution to the considered task. According to the method, the solution can be carried out through the following algorithm:

1. Mapping of the three-dimensional initial conditions of the task of spatial spline construction on projection planes $\pi_{1}$ and $\pi_{2}$.

2. Construction of the two flat splines that constitute the orthogonal projections of the sought spline and form its model.

3. Reconstruction of the geometry, including shape and differential geometric characteristics, of the spatial spline by its model.

4. Verification of the acquired model of spatial spline formation.

There is a reverse task of the Monge method known in the field of engineering geometry: spatial reconstruction of the shape and the internal structure of a 3D object by its orthogonal projections, i.e. by a drawing. There is no complete solution to the task regardless of its practical relevance [10]. With regard for the application of a spline as the universal computational instrument in the problems of formation, it is logical to consider relevant the problem of construction of a spline by its orthogonal projection images. 


\section{Problem Definition}

There are projections of boundary points $\mathrm{n}$ of polynomial segments given on a Monge model. The projections of the boundary conditions, namely vector derivatives of the initial orders in the initial point of the first segment and the terminal point of the final segment, are also given. It is required to construct a spline of $\mathrm{n}$ polynomial segments connected under the order of smoothness $C^{k}$.

\section{Spatial Spline Projectional Construction}

Formation of a spatial spline is based on projectional formation of its segments with consideration for the given smoothness $C^{k}$ of connection of its segments and the specified boundary conditions in the form of projections of the initial vector derivatives in the initial point of the first segment and in the terminal point of the final segment. The order of smoothness and the boundary conditions must define a complete system of linear equations, the solution to which yields the missing boundary conditions in the points of connection of the segments.

\subsection{Spatial Polynomial Segment Projectional Construction}

A segment is defined by a pair of points and boundary conditions in the form of the initial vector derivatives. The order $n$ of the polynomial defining a segment depends on the number of boundary conditions: $n=k_{1}+k_{2}+1$, where $k_{1}$ and $k_{2}$ represent the number of the boundary conditions in the first and the second respective points.

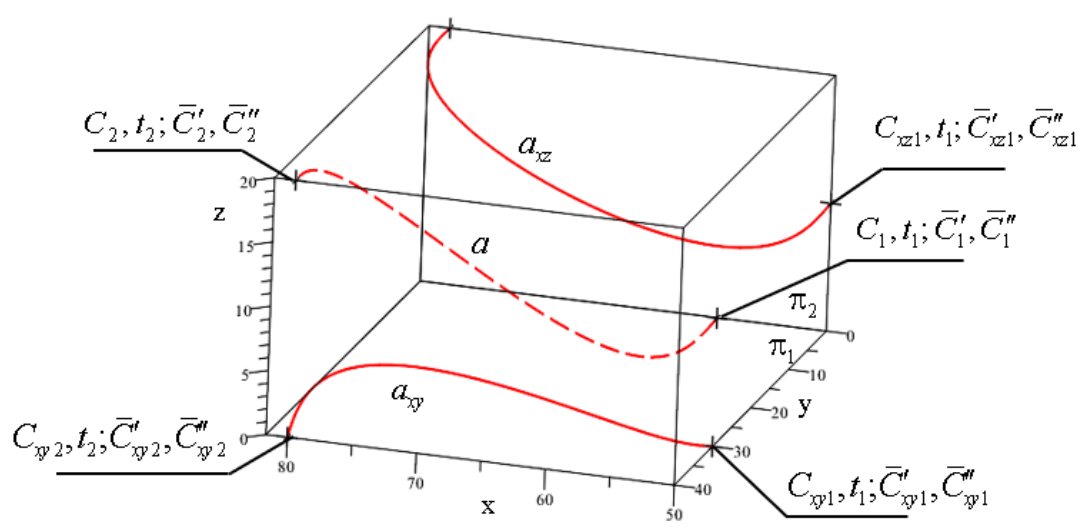

Fig. 1. Constructed orthogonal projections $a_{x y}$ and $a_{x z}$ of the sought polynomial segment $a$

Let us consider the problem of spatial polynomial segment construction given the orthogonal projections of its points and boundary conditions. 
A polynomial segment passes through points $C_{1}$ and $C_{2}$. The points $C_{1}, C_{2}$ are defined by respective projections $\bar{C}_{x y 1}\left(x_{1}, y_{1}\right), \bar{C}_{x y 2}\left(x_{2}, y_{2}\right)$ on $\pi_{1}, \bar{C}_{x z 1}\left(x_{1}, z_{1}\right), \bar{C}_{x z 2}\left(x_{2}, z_{2}\right)$ on $\pi_{2}$. Projections of boundary conditions in these points are also given: $\bar{C}_{x y 1}^{\prime}, \bar{C}_{x y 1}^{\prime \prime}$, $\bar{C}_{x z 1}^{\prime}, \bar{C}_{x z 1}^{\prime \prime}$ and $\bar{C}_{x y 2}^{\prime}, \bar{C}_{x y 2}^{\prime \prime}, \bar{C}_{x z 2}^{\prime}, \bar{C}_{x z 2}^{\prime \prime}$ in projection planes $\pi_{1}$ and $\pi_{2}$ respectively (see Fig.1). $\bar{C}_{x y 1}^{\prime}, \bar{C}_{x z 1}^{\prime}, \bar{C}_{x y 2}^{\prime}, \bar{C}_{x z 2}^{\prime}$ are the first vector derivatives constituting projections of tangent vectors in endpoints of the sought segment a; $\bar{C}_{x y 1}^{\prime \prime}, \bar{C}_{x z 1}^{\prime \prime}, \bar{C}_{x y 2}^{\prime \prime}, \bar{C}_{x z 2}^{\prime \prime}$ are the second vector derivatives constituting projections of acceleration vectors of a point passing along the segment $a$.

It should be noted given the initial conditions that the projections of the sought segment can be described by a polynomial $\bar{a}(t)=\sum_{i=1}^{6} \bar{A}_{i} t^{i-1}$ :

$$
\begin{aligned}
& \bar{a}_{x y}(t)=\bar{A}_{x y 1}+\bar{A}_{x y 2} t+\bar{A}_{x y 3} t^{2}+\bar{A}_{x y 4} t^{3}+\bar{A}_{x y 5} t^{4}+\bar{A}_{x y 6} t^{5}, t_{1} \leq t \leq t_{2} . \\
& \bar{a}_{x z}(t)=\bar{A}_{x z 1}+\bar{A}_{x z 2} t+\bar{A}_{x z 3} t^{2}+\bar{A}_{x z z} t^{3}+\bar{A}_{x z} t^{4}+\bar{A}_{x z 6} t^{5}, t_{1} \leq t \leq t_{2} .
\end{aligned}
$$

The initial conditions are sufficient to determine vector coefficients $\bar{A}_{x y i}$ and $\bar{A}_{x z i}$ $i=1,2, \ldots, 6$ in equations (2) and (3). Let us begin by expressing the first and the second derivatives of vector functions $\bar{a}_{x y}(t)$ and $\bar{a}_{x z}(t)$ :

$$
\begin{array}{ll}
\bar{a}_{x y}^{\prime}(t)=\sum_{i=2}^{6} \bar{A}_{x y i}(i-1) t^{i-2}, \quad \bar{a}_{x y}^{\prime \prime}(t)=\sum_{i=3}^{6} \bar{A}_{x y i}(i-1)(i-2) t^{i-3} . \\
\bar{a}_{x z}^{\prime}(t)=\sum_{i=2}^{6} \bar{A}_{x z i}(i-1) t^{i-2}, \quad \bar{a}_{x z}^{\prime \prime}(t)=\sum_{i=3}^{6} \bar{A}_{x z i}(i-1)(i-2) t^{i-3} .
\end{array}
$$

Without loss of generality we can accept that $t_{1}=0$, i.e. $0 \leq t \leq t_{2}$. This allows us to determine the values of the first three vector coefficients in equations (2) and (3):

$$
\begin{aligned}
& \bar{a}_{x y}(0)=\bar{A}_{x y 1}=\bar{C}_{x y 1}, \quad \bar{a}_{x y}^{\prime}(0)=\bar{A}_{x y 2}=\bar{C}_{x y 1}^{\prime}, \\
& \bar{a}_{x y}^{\prime \prime}(0)=2 \bar{A}_{x y 3}=\bar{C}_{x y 1}^{\prime \prime}, \text { i.e. } \bar{A}_{x y 3}=\frac{1}{2} \bar{C}_{x y 1}^{\prime \prime} . \\
& \bar{a}_{x z}(0)=\bar{A}_{x z 1}=\bar{C}_{x z 1}, \quad \bar{a}_{x z}^{\prime}(0)=\bar{A}_{x z 2}=\bar{C}_{x z 1}^{\prime}, \\
& \bar{a}_{x z}^{\prime \prime}(0)=2 \bar{A}_{x z 3}=\bar{C}_{x z 1}^{\prime \prime}, \text { i.e. } \bar{A}_{x z 3}=\frac{1}{2} \bar{C}_{x z 1}^{\prime \prime} .
\end{aligned}
$$

The remaining three vector coefficients in equations (2) and (3) are determined through the following conditions: 


$$
\begin{array}{ll}
\bar{a}_{x y}\left(t_{2}\right)=\bar{C}_{x y 2}, & \bar{a}_{x y}^{\prime}\left(t_{2}\right)=\bar{C}_{x y 2}^{\prime}, \quad \bar{a}_{x y}^{\prime \prime}\left(t_{2}\right)=\bar{C}_{x y 2}^{\prime \prime}, \\
\bar{a}_{x z}\left(t_{2}\right)=\bar{C}_{x z 2}, & \bar{a}_{x z}^{\prime}\left(t_{2}\right)=\bar{C}_{x z 2}^{\prime}, \quad \bar{a}_{x z}^{\prime \prime}\left(t_{2}\right)=\bar{C}_{x z 2}^{\prime \prime},
\end{array}
$$

that result in systems of equations

$$
\left\{\begin{array} { l } 
{ \sum _ { i = 1 } ^ { 6 } \overline { A } _ { x y i } t ^ { i - 1 } - \overline { C } _ { x y 2 } = 0 , } \\
{ \sum _ { i = 2 } ^ { 6 } \overline { A } _ { x y i } ( i - 1 ) t ^ { i - 2 } - \overline { C } _ { x y 2 } ^ { \prime } = 0 , } \\
{ \sum _ { i = 3 } ^ { 6 } \overline { A } _ { x y i } ( i - 1 ) ( i - 2 ) t ^ { i - 3 } - \overline { C } _ { x y 2 } ^ { \prime \prime } = 0 , }
\end{array} \quad \left\{\begin{array}{l}
\sum_{i=1}^{6} \bar{A}_{x z i} t^{i-1}-\bar{C}_{x z 2}=0, \\
\sum_{i=2}^{6} \bar{A}_{x z i}(i-1) t^{i-2}-\bar{C}_{x z 2}^{\prime}=0, \\
\sum_{i=3}^{6} \bar{A}_{x z i}(i-1)(i-2) t^{i-3}-\bar{C}_{x z 2}^{\prime \prime}=0 .
\end{array}\right.\right.
$$

The systems of equations (9) yield the values of the coefficients $\bar{A}_{x y 4}, \bar{A}_{x y 5}, \bar{A}_{x y 6}$ and $\bar{A}_{x z 4}, \bar{A}_{x z 5}, \bar{A}_{x z 6}$.

The systems of equations (9) can be presented in matrix form:

$$
\begin{aligned}
& {\left[\bar{A}_{x y}\right]=\left[\begin{array}{c}
\bar{A}_{x y 1} \\
\bar{A}_{x y 2} \\
\bar{A}_{x y 3} \\
\bar{A}_{x y 4} \\
\bar{A}_{x y 5} \\
\bar{A}_{x y 6}
\end{array}\right]=\left[\begin{array}{cccccc}
1 & 0 & 0 & 0 & 0 & 0 \\
0 & 1 & 0 & 0 & 0 & 0 \\
0 & 0 & \frac{1}{2} & 0 & 0 & 0 \\
-\frac{10}{t_{2}^{3}} & -\frac{6}{t_{2}^{2}} & -\frac{1.5}{t_{2}} & \frac{10}{t_{2}^{3}} & -\frac{4}{t_{2}^{2}} & \frac{0.5}{t_{2}} \\
\frac{15}{t_{2}^{4}} & \frac{8}{t_{2}^{3}} & \frac{1.5}{t_{2}^{2}} & -\frac{15}{t_{2}^{4}} & \frac{7}{t_{2}^{3}} & -\frac{1}{t_{2}^{2}} \\
-\frac{6}{t_{2}^{5}} & -\frac{3}{t_{2}^{4}} & -\frac{0.5}{t_{2}^{3}} & \frac{6}{t_{2}^{5}} & -\frac{3}{t_{2}^{4}} & \frac{0.5}{t_{2}^{3}}
\end{array}\right]\left[\begin{array}{l}
\bar{C}_{x y 1} \\
\bar{C}_{x y 11}^{\prime} \\
\bar{C}_{x y 1}^{\prime \prime} \\
\bar{C}_{x y 2} \\
\bar{C}_{x y 2}^{\prime} \\
\bar{C}_{x y 2}^{\prime \prime}
\end{array}\right] .} \\
& {\left[\bar{A}_{x z}\right]=\left[\begin{array}{c}
\bar{A}_{x z 1} \\
\bar{A}_{x z 2} \\
\bar{A}_{x z 3} \\
\bar{A}_{x z 4} \\
\bar{A}_{x z 5} \\
\bar{A}_{x z 6}
\end{array}\right]=\left[\begin{array}{cccccc}
1 & 0 & 0 & 0 & 0 & 0 \\
0 & 1 & 0 & 0 & 0 & 0 \\
0 & 0 & \frac{1}{2} & 0 & 0 & 0 \\
-\frac{10}{t_{2}^{3}} & -\frac{6}{t_{2}^{2}} & -\frac{1.5}{t_{2}} & \frac{10}{t_{2}^{3}} & -\frac{4}{t_{2}^{2}} & \frac{0.5}{t_{2}} \\
\frac{15}{t_{2}^{4}} & \frac{8}{t_{2}^{3}} & \frac{1.5}{t_{2}^{2}} & -\frac{15}{t_{2}^{4}} & \frac{7}{t_{2}^{3}} & -\frac{1}{t_{2}^{2}} \\
-\frac{6}{t_{2}^{5}} & -\frac{3}{t_{2}^{4}} & -\frac{0.5}{t_{2}^{3}} & \frac{6}{t_{2}^{5}} & -\frac{3}{t_{2}^{4}} & \frac{0.5}{t_{2}^{3}}
\end{array}\right]\left[\begin{array}{l}
\bar{C}_{x z 1} \\
\bar{C}_{x z 1}^{\prime} \\
\bar{C}_{x z 1}^{\prime \prime} \\
\bar{C}_{x z 2} \\
\bar{C}_{x z 2}^{\prime} \\
\bar{C}_{x z 2}^{\prime \prime}
\end{array}\right] .}
\end{aligned}
$$


The matrices (10) and (11) are of explicit form and can be expressed in a contracted form: $[\bar{A}]=[T] \cdot[G]$, where $[T]$ and $[G]$ represent parameter matrix and derivative matrix respectively.

By substituting the values of vector coefficients $(10,11)$ into equations (2) and (3), we acquire the sought equations of polynomial curve segment projections in projection planes $\pi_{1}$ and $\pi_{2}$. The two projections (2) and (3) define, as it follows from the properties of Monge drawing, the shape and the differential geometric characteristics of a polynomial segment in space.

\subsection{Spatial Spline Construction}

Let us specify the projections of boundary points of a finite number of polynomial segments, e.g., three segments $\left(C_{x y 1}, C_{x z 1}\right),\left(C_{x y 2}, C_{x z 2}\right),\left(C_{x y 3}, C_{x z 3}\right),\left(C_{x y 4}, C_{x z 4}\right)$ on a Monge drawing. Let us also specify the projections of the boundary conditions, the vector derivatives of the first and the second order in the initial point of the first and the terminal point of the third segment (see Fig.2).



Fig. 2. The initial data for projectional construction of a spatial spline consisting of three polynomial segments.

Let us construct projections of the spline in projection planes $\pi_{1}$ and $\pi_{2}$. Each projection consists of three segments $a_{x y i}$ and $a_{x z i}, i=1,2,3$ connected under the order of smoothness $C^{3}$. In order to connect the segments by three in projection planes $\pi_{1}$ and $\pi_{2}$ under the order of smoothness $C^{3}$ it is required to establish equality of the second and the third derivatives of vector-functions in the points of connection: 


$$
\left\{\begin{array} { l } 
{ \overline { a } _ { x y i } ^ { \prime \prime } ( t = 1 ) = \overline { a } _ { x y i + 1 } ^ { \prime \prime } ( t = 0 ) , } \\
{ \overline { a } _ { x y i } ^ { \prime \prime \prime } ( t = 1 ) = \overline { a } _ { x y i + 1 } ^ { \prime \prime \prime } ( t = 0 ) , }
\end{array} \left\{\begin{array}{l}
\bar{a}_{x z i}^{\prime \prime}(t=1)=\bar{a}_{x z i+1}^{\prime \prime}(t=0), \\
\bar{a}_{x z i}^{\prime \prime \prime}(t=1)=\bar{a}_{x z i+1}^{\prime \prime \prime}(t=0),
\end{array}\right.\right.
$$

where $i=1,2 ; 0 \leq t \leq 1$.

The system of linear equations (12) allows us to determine the unknown vectors of the first and the second derivatives in connection points of the segments in projection plane $\pi_{1}$ :

$$
\begin{aligned}
& \bar{C}_{x y 2}^{\prime}=-\frac{8}{3} \bar{C}_{x y 1}^{\prime}+\frac{8}{3} \bar{C}_{x y 3}^{\prime} \frac{1}{3} \bar{C}_{x y 1}^{\prime \prime}+\frac{2}{3} \bar{C}_{x y 3}^{\prime \prime}-2\left(\frac{1}{3} \bar{C}_{x y 1}-\frac{20}{3} \bar{C}_{x y 2}+\frac{10}{3} \bar{C}_{x y 3}\right), \\
& \bar{C}_{x y 2}^{\prime \prime}=\frac{4}{3} \bar{C}_{x y 1}^{\prime}-\frac{4}{3} \bar{C}_{x y 3}^{\prime}+\frac{1}{6} \bar{C}_{x y 1}^{\prime \prime}+\frac{1}{6} \bar{C}_{x y 3}^{\prime \prime}+\frac{10}{3} \bar{C}_{x y 1}-\frac{20}{3} \bar{C}_{x y 2}+\frac{10}{3} \bar{C}_{x y 3}, \\
& \bar{C}_{x y 3}^{\prime}=-\frac{8}{3} \bar{C}_{x y 2}^{\prime}+\frac{8}{3} \bar{C}_{x y 4}^{\prime}-\frac{1}{3} \bar{C}_{x y 2}^{\prime \prime}+\frac{2}{3} \bar{C}_{x y 4}^{\prime \prime}-2\left(\frac{10}{3} \bar{C}_{x y 2}-\frac{20}{3} \bar{C}_{x y 3}+\frac{10}{3} \bar{C}_{x y 4}\right), \\
& \bar{C}_{x y 3}^{\prime \prime}=\frac{4}{3} \bar{C}_{x y 2}^{\prime}-\frac{4}{3} \bar{C}_{x y 4}^{\prime}+\frac{1}{6} \bar{C}_{x y 2}^{\prime \prime}+\frac{1}{6} \bar{C}_{x y 4}^{\prime \prime}+\frac{10}{3} \bar{C}_{x y 2}-\frac{20}{3} \bar{C}_{x y 3}+\frac{10}{3} \bar{C}_{x y 4},
\end{aligned}
$$

and in projection plane $\pi_{2}$ :

$$
\begin{aligned}
& \bar{C}_{x z 2}^{\prime}=-\frac{8}{3} \bar{C}_{x z 1}^{\prime}+\frac{8}{3} \bar{C}_{x z 3}^{\prime} \frac{1}{3} \bar{C}_{x z 1}^{\prime \prime}+\frac{2}{3} \bar{C}_{x z 3}^{\prime \prime}-2\left(\frac{1}{3} \bar{C}_{x z 1}-\frac{20}{3} \bar{C}_{x z 2}+\frac{10}{3} \bar{C}_{x z 3}\right), \\
& \bar{C}_{x z 2}^{\prime \prime}=\frac{4}{3} \bar{C}_{x z 1}^{\prime}-\frac{4}{3} \bar{C}_{x z 3}^{\prime}+\frac{1}{6} \bar{C}_{x z 1}^{\prime \prime}+\frac{1}{6} \bar{C}_{x z 3}^{\prime \prime}+\frac{10}{3} \bar{C}_{x z 1}-\frac{20}{3} \bar{C}_{x z 2}+\frac{10}{3} \bar{C}_{x z 3}, \\
& \bar{C}_{x z 3}^{\prime}=-\frac{8}{3} \bar{C}_{x z 2}^{\prime}+\frac{8}{3} \bar{C}_{x z 4}^{\prime}-\frac{1}{3} \bar{C}_{x z 2}^{\prime \prime}+\frac{2}{3} \bar{C}_{x z 4}^{\prime \prime}-2\left(\frac{10}{3} \bar{C}_{x z 2}-\frac{20}{3} \bar{C}_{x z 3}+\frac{10}{3} \bar{C}_{x z 4}\right), \\
& \bar{C}_{x z 3}^{\prime \prime}=\frac{4}{3} \bar{C}_{x z 2}^{\prime}-\frac{4}{3} \bar{C}_{x z 4}^{\prime}+\frac{1}{6} \bar{C}_{x z 2}^{\prime \prime}+\frac{1}{6} \bar{C}_{x z 4}^{\prime \prime}+\frac{10}{3} \bar{C}_{x z 2}-\frac{20}{3} \bar{C}_{x z 3}+\frac{10}{3} \bar{C}_{x z 4} .
\end{aligned}
$$

Thus we have determined the required vector derivatives in points of connection under the order of smoothness $C^{3}$ are in planes $\pi_{1}$ and $\pi_{2}$. This allows us to move to the equations of the segments in the form of polynomials (2) and (3) through the equations (10) and (11).

The properties of the Monge drawing - algebraic curve order preservation and drawing reversibility - allow us to conclude that a spline is reconstructible by its two orthogonal projections. Transformation of the equations (13) and (14) results in systems of linear equations with the unknown vectors $\bar{C}_{x y 2}^{\prime}, \bar{C}_{x y 3}^{\prime}, \bar{C}_{x y 2}^{\prime \prime}, \bar{C}_{x y 3}^{\prime \prime}$ and $\bar{C}_{x z 2}^{\prime}$, $\bar{C}_{x z 3}^{\prime}, \bar{C}_{x z 2}^{\prime \prime}, \bar{C}_{x z 3}^{\prime \prime}$ relocated to the left parts of the equations: 


$$
\begin{aligned}
& \left\{\begin{array}{l}
-\bar{C}_{x y 2}^{\prime}+\frac{8}{3} \bar{C}_{x y 3}^{\prime}+\frac{2}{3} \bar{C}_{x y 3}^{\prime \prime}=2\left(\frac{10}{3} \bar{C}_{x y 1}-\frac{20}{3} \bar{C}_{x y 2}+\frac{10}{3} \bar{C}_{x y 3}+\frac{4}{3} \bar{C}_{x y 1}^{\prime}+\frac{1}{6} \bar{C}_{x y 1}^{\prime \prime}\right), \\
\frac{4}{3} \bar{C}_{x y 3}^{\prime}+\bar{C}_{x y 2}^{\prime \prime}-\frac{1}{6} \bar{C}_{x y 3}^{\prime \prime}=\frac{10}{3} \bar{C}_{x y 1}-\frac{20}{3} \bar{C}_{x y 2}+\frac{10}{3} \bar{C}_{x y 3}+\frac{4}{3} \bar{C}_{x y 1}^{\prime}+\frac{1}{6} \bar{C}_{x y 1}^{\prime \prime}, \\
-\frac{8}{3} \bar{C}_{x y 2}^{\prime}-\bar{C}_{x y 3}^{\prime}+\frac{1}{3} \bar{C}_{x y 2}^{\prime \prime}=2\left(\frac{10}{3} \bar{C}_{x y 2}-\frac{20}{3} \bar{C}_{x y 3}+\frac{10}{3} \bar{C}_{x y 4}-\frac{4}{3} \bar{C}_{x y 4}^{\prime}-\frac{1}{3} \bar{C}_{x y 4}^{\prime \prime}\right), \\
-\frac{4}{3} \bar{C}_{x y 2}^{\prime}-\frac{1}{6} \bar{C}_{x y 2}^{\prime \prime}+\bar{C}_{x y 3}^{\prime \prime}=\frac{10}{3} \bar{C}_{x y 2}-\frac{20}{3} \bar{C}_{x y 3}+\frac{10}{3} \bar{C}_{x y 4}-\frac{4}{3} \bar{C}_{x y 4}^{\prime}+\frac{1}{6} \bar{C}_{x y 4}^{\prime \prime} .
\end{array}\right. \\
& \left\{\begin{array}{l}
-\bar{C}_{x z 2}^{\prime}+\frac{8}{3} \bar{C}_{x z 3}^{\prime}+\frac{2}{3} \bar{C}_{x z 3}^{\prime \prime}=2\left(\frac{10}{3} \bar{C}_{x z 1}-\frac{20}{3} \bar{C}_{x z 2}+\frac{10}{3} \bar{C}_{x z 3}+\frac{4}{3} \bar{C}_{x z 1}^{\prime}+\frac{1}{6} \bar{C}_{x z 1}^{\prime \prime}\right), \\
\frac{4}{3} \bar{C}_{x z 3}^{\prime}+\bar{C}_{x z 2}^{\prime \prime}-\frac{1}{6} \bar{C}_{x z 3}^{\prime \prime}=\frac{10}{3} \bar{C}_{x z 1}-\frac{20}{3} \bar{C}_{x z 2}+\frac{10}{3} \bar{C}_{x z 3}+\frac{4}{3} \bar{C}_{x z 1}^{\prime}+\frac{1}{6} \bar{C}_{x z 1}^{\prime \prime}, \\
-\frac{8}{3} \bar{C}_{x z 2}^{\prime}-\bar{C}_{x z 3}^{\prime}+\frac{1}{3} \bar{C}_{x z 2}^{\prime \prime}=2\left(\frac{10}{3} \bar{C}_{x z 2}-\frac{20}{3} \bar{C}_{x z 3}+\frac{10}{3} \bar{C}_{x z 4}-\frac{4}{3} \bar{C}_{x z 4}^{\prime}-\frac{1}{3} \bar{C}_{x z 4}^{\prime \prime}\right), \\
-\frac{4}{3} \bar{C}_{x z 2}^{\prime}-\frac{1}{6} \bar{C}_{x z 2}^{\prime \prime}+\bar{C}_{x z 3}^{\prime \prime}=\frac{10}{3} \bar{C}_{x z 2}-\frac{20}{3} \bar{C}_{x z 3}+\frac{10}{3} \bar{C}_{x z 4}-\frac{4}{3} \bar{C}_{x z 4}^{\prime}+\frac{1}{6} \bar{C}_{x z 4}^{\prime \prime} .
\end{array}\right.
\end{aligned}
$$

Let us express the equations $(15,16)$ in the matrix form. It is convenient to apply the matrix form in calculation of the unknown vector coefficients through one of the methods of solution of simultaneous linear algebraic equations known in the field of linear algebra, for example, through the Jordan-Gauss method:

$$
\begin{aligned}
& {\left[\begin{array}{cccc}
-1 & \frac{8}{3} & 0 & \frac{2}{3} \\
0 & \frac{4}{3} & 1 & -\frac{1}{6} \\
-\frac{8}{3} & -1 & \frac{1}{3} & 0 \\
-\frac{4}{3} & 0 & -\frac{1}{6} & 1
\end{array}\right]\left[\begin{array}{l}
\bar{C}_{x y 2}^{\prime} \\
\bar{C}_{x y 3}^{\prime} \\
\bar{C}_{x y 2}^{\prime \prime} \\
\bar{C}_{x y 3}^{\prime \prime}
\end{array}\right]=\left[\begin{array}{l}
2\left(\frac{10}{3} \bar{C}_{x y 1}-\frac{20}{3} \bar{C}_{x y 2}+\frac{10}{3} \bar{C}_{x y 3}+\frac{4}{3} \bar{C}_{x y 1}^{\prime}+\frac{1}{6} \bar{C}_{x y 1}^{\prime \prime}\right) \\
\frac{10}{3} \bar{C}_{x y 1}-\frac{20}{3} \bar{C}_{x y 2}+\frac{10}{3} \bar{C}_{x y 3}+\frac{4}{3} \bar{C}_{x y 1}^{\prime}+\frac{1}{6} \bar{C}_{x y 1}^{\prime \prime} \\
2\left(\frac{10}{3} \bar{C}_{x y 2}-\frac{20}{3} \bar{C}_{x y 3}+\frac{10}{3} \bar{C}_{x y 4}-\frac{4}{3} \bar{C}_{x y 4}^{\prime}-\frac{1}{3} \bar{C}_{x y 4}^{\prime \prime}\right) \\
\frac{10}{3} \bar{C}_{x y 2}-\frac{20}{3} \bar{C}_{x y 3}+\frac{10}{3} \bar{C}_{x y 4}-\frac{4}{3} \bar{C}_{x y 4}^{\prime}+\frac{1}{6} \bar{C}_{x y 4}^{\prime \prime}
\end{array}\right],} \\
& {\left[\begin{array}{cccc}
-1 & \frac{8}{3} & 0 & \frac{2}{3} \\
0 & \frac{4}{3} & 1 & -\frac{1}{6} \\
-\frac{8}{3} & -1 & \frac{1}{3} & 0 \\
-\frac{4}{3} & 0 & -\frac{1}{6} & 1
\end{array}\right]\left[\begin{array}{l}
\bar{C}_{x z 2}^{\prime} \\
\bar{C}_{x z 3}^{\prime} \\
\bar{C}_{x z 2}^{\prime \prime} \\
\bar{C}_{x z 3}^{\prime \prime}
\end{array}\right]=\left[\begin{array}{l}
2\left(\frac{10}{3} \bar{C}_{x z 1}-\frac{20}{3} \bar{C}_{x z 2}+\frac{10}{3} \bar{C}_{x z 3}+\frac{4}{3} \bar{C}_{x z 1}^{\prime}+\frac{1}{6} \bar{C}_{x z 1}^{\prime \prime}\right) \\
\frac{10}{3} \bar{C}_{x z 1}-\frac{20}{3} \bar{C}_{x z 2}+\frac{10}{3} \bar{C}_{x z 3}+\frac{4}{3} \bar{C}_{x z 1}^{\prime}+\frac{1}{6} \bar{C}_{x z 1}^{\prime \prime} \\
2\left(\frac{10}{3} \bar{C}_{x z 2}-\frac{20}{3} \bar{C}_{x z 3}+\frac{10}{3} \bar{C}_{x z 4}-\frac{4}{3} \bar{C}_{x z 4}^{\prime}-\frac{1}{3} \bar{C}_{x z 4}^{\prime \prime}\right) \\
\frac{10}{3} \bar{C}_{x z 2}-\frac{20}{3} \bar{C}_{x z 3}+\frac{10}{3} \bar{C}_{x z 4}-\frac{4}{3} \bar{C}_{x z 4}^{\prime}+\frac{1}{6} \bar{C}_{x z 4}^{\prime \prime}
\end{array}\right]}
\end{aligned}
$$




\subsection{Numerical Experiment}

Let us consider an example. Given the boundary conditions of three segments $C_{x y 1}(0,20), \quad C_{x y 2}(20,30), \quad C_{x y 3}(50,30), C_{x y 4}(80,40) ; \quad C_{x z 1}(0,10), C_{x z 2}(20,20)$, $C_{x z 3}(50,40), \quad C_{x z 4}(80,60) ; \quad \bar{C}_{x y 1}^{\prime}(1,3), \quad \bar{C}_{x y 1}^{\prime \prime}(-1,1), \quad \bar{C}_{x z 1}^{\prime}(1,1), \quad \bar{C}_{x z 1}^{\prime \prime}(-1,2)$; $\bar{C}_{x y 4}^{\prime}(-17,85), \quad \bar{C}_{x y 4}^{\prime \prime}(-1,-40), \bar{C}_{x z 4}^{\prime}(-17,18), \bar{C}_{x z 4}^{\prime \prime}(-1,-7)$, it is required to construct a spatial three-segment spline, segments of which are connected under the order of smoothness $C^{3}$ (see Fig. 3).

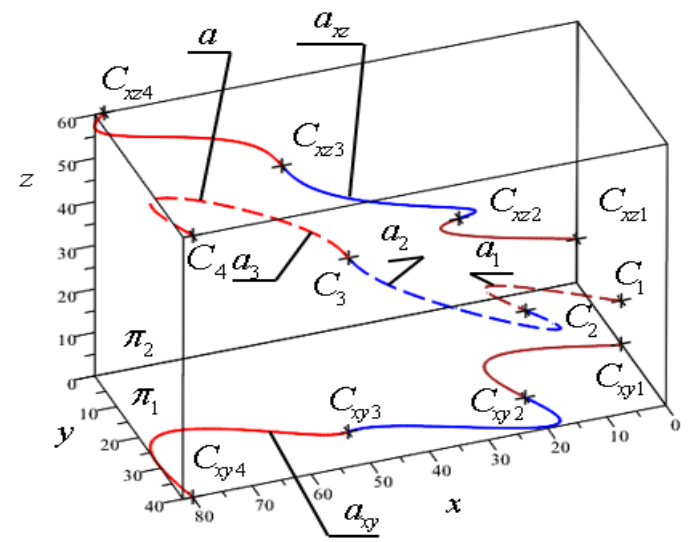

Fig. 3. A three-segment spline a reconstructed by its orthogonal projections $a_{x y}$ and $a_{x z}$.

As a result of calculations performed using the equations (15), (16), (10), and (11), a three-segment spline $a: a=a_{1} \cup a_{2} \cup a_{3}$ is acquired in space by its orthogonal projections. Each of the segments is defined by a polynomial of the fifth order. Table 1 represents the values of curvature and torsion in the points of connection of the spline $a$. The calculation of the values of curvature $k$ and torsion $\chi$ in the points of the spatial spline modeled by a pair of projections are performed through the known formulas [7]

$$
\begin{gathered}
k=\sqrt{A^{2}+B^{2}+C^{2}} . \\
\chi=\frac{(\alpha \sigma-\beta \rho)[(B L-C K) D+(A K-F B) E]}{k^{2}}
\end{gathered}
$$

where $\quad \alpha=\bar{\tau}_{1} \cdot \bar{i}, \quad \beta=\bar{v}_{1} \cdot \bar{i}, \quad \gamma=\bar{\tau}_{2} \cdot \bar{i}, \quad \delta=\bar{v}_{2} \cdot \bar{i}, \quad \varepsilon=\bar{\tau}_{2} \cdot \bar{k}, \quad \eta=\bar{v}_{2} \cdot \bar{k}$, $\frac{d s_{1}}{d s}=\frac{|\gamma|}{\sqrt{\alpha^{2} \varepsilon^{2}+\gamma^{2}}} ; \frac{d s_{2}}{d s}=\frac{|\alpha|}{\sqrt{\alpha^{2} \varepsilon^{2}+\gamma^{2}}}, \rho=\bar{\tau}_{1} \cdot \bar{j}, \sigma=\overline{v_{1}} \cdot \bar{j}$ (see Fig. 4).

Calculation of the values of the rest of the coefficients of the formulas (19) and (20) is omitted due to its bulkiness. However, these values are defined by geometry 
and differential characteristics of the given projections $a_{1}$ and $a_{2}$ of the reconstructed spatial curve $a$ (see Fig. 4). The formulas for the coefficients are detailed in [7].

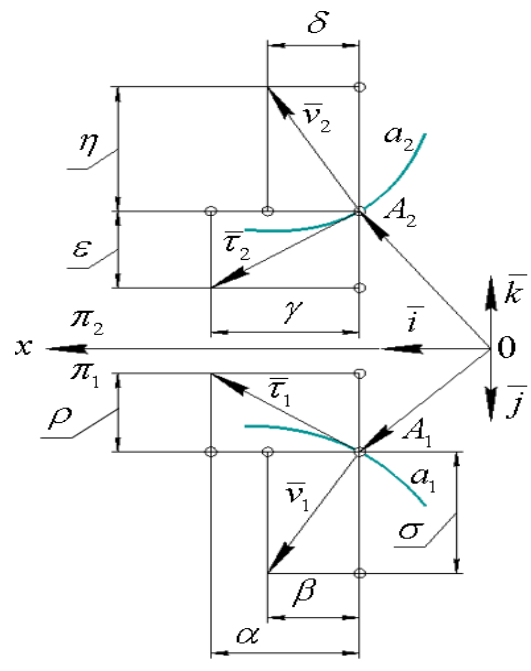

Fig. 4. Projections a1 and $a_{2}$ of the spatial curve $a$ and its tangent and normal unit vectors in point $A$.

Table 1. Curvature and torque values in boundary points of segments $a_{i}, i=1,2,3$.

\begin{tabular}{|l|l|l|l|}
\hline Segment & Boundary point & Curvature value & Torque value \\
\hline \multirow{2}{*}{ segment $a_{1}: a_{1}(t)$} & $C_{1}, t=0$ & 0.1938188331 & 265.2382716 \\
\cline { 2 - 4 } & $C_{2}, t=1$ & 0.003720362183 & -0.6729237999 \\
\hline \multirow{2}{*}{ segment $a_{2}: a_{2}(t)$} & $C_{2}, t=0$ & 0.003720362183 & -0.6729237999 \\
\cline { 2 - 4 } & $C_{3}, t=1$ & 0.01329625195 & 1.898108432 \\
\hline \multirow{2}{*}{ segment $a_{3}: a_{3}(t)$} & $C_{3}, t=0$ & 0.01329625195 & 1.898108433 \\
\cline { 2 - 4 } & $C_{4}, t=1$ & 0.001134369327 & 0.7192054044 \\
\hline
\end{tabular}

\section{Conclusion}

The solution to the problem of spatial spline construction with the initial conditions given on the Monge model is considered and confirmed on example of the presented numerical solution. The considered problem is classified as reverse problem of engineering geometry performed on the Monge model. Its solution can be applied in reconstruction of one-dimensional geometric forms for surface form generation of computer 3D geometric models of objects by their blueprints. The properties of the Monge model allow for projectional solution to the problem of spatial spline construction regardless of its type (including rational and fractionally rational Bezier splines [8,11], B-splines, etc.) and order of its equations. 


\section{References}

1. Agoston, M.K.: Computer Graphics and Geometric Modeling. Implementation and Algorithms. Springer Verlag London Limited (2005).

2. Farin, G.: Curves and Surfaces for Computer Aided Geometric Design. Implementation and Algorithms, A Practical Guide. Academic Press (1990).

3. Farouki, R.T.: Pythagorean - Hodograph Curves. Algebra and Geometry Inseparable. Springer, Verlag Berlin Heidelberg. (2008).

4. Gallier, J.: Curves and Surfaces in Geometric Modeling: Theory and Algorithms. University of Pennsylvania, USA Philadelphia (2018).

5. Handbook of Computer Aided Geometric Design. In: Farin, G., Hoschek, J. (eds.) Elsevier, Amsterdam (2002).

6. Ivanov, G.S.: Teoreticheskie osnovy nachertatel'noi geometrii [Theoretical foundations of descriptive geometry]. Mashinostroenie Publ., Moscow (1998).

7. Panchuk, K.L., Yurkov, V.Yu., Kaygorodtseva, N.V.: Matematicheskie osnovy` geometricheskogo modelirovaniya krivy`x linij [Mathematical foundations of geometric modeling of curved lines]. Omsk State Technical University, Omsk (2020).

8. Salomon, D.: Curves and Surfaces for Computer Graphics. Springer, Verlag New York (2006).

9. Shikin, E.V., Plis A.I.: Krivy`e i poverxnosti na e`krane komp`yutera: Ruk. po splajnam dlya pol zovatelej [Curves and surfaces on a computer screen: Hands. on splines for users]. Dialog-MEPhI, Moscow (1996).

10. Moshkova, T.V., Rotkov, S.I., Tyurina, V.A.: The problem of the synthesis of a $3 \mathrm{~d}$ model of an object from its projection images. Analytical review. Scientific visualization, vol. 10(1), pp. 135-156. National Research Nuclear University MEPhI, Moscow (2018). DOI: 10.26583/sv.10.1.11.

11. Panchuk, K. L., Myasoedova, T. M., Odinets, M. N.: Construction of a discrete planar contour by fractional rational Bezier curves of second order. Journal of Physics: Conference Series 1441, (2020). DOI:10.1088/1742-6596/1441/1/012072. 